\title{
A Primer on Energy-Efficient Synchronization of WSN Nodes over Correlated Rayleigh Fading Channels
}

\author{
Pablo Briff, Student Member, IEEE, Ariel Lutenberg, Member, IEEE, Leonardo Rey Vega, \\ Fabian Vargas, Member, IEEE, and Mohammad Patwary, Senior Member, IEEE
}

\begin{abstract}
In this paper we propose a lower bound on the energy required for synchronizing nodes in a Wireless Sensor Network (WSN) by using statistical estimation techniques. The energy required to synchronize a pair of nodes within a network with predefined synchronization accuracy is modelled as function of the transceivers power and the number of transmitted messages. In our analysis, we have considered the dynamic nature of nodes communicating within a correlated Rayleigh fading channel. A unified mathematical model has been defined to analyze the impact of nodes' relative velocity on the pairwise energy and synchronization accuracy trade-off.
\end{abstract}

Index Terms-Wireless sensor networks, clock synchronization, outage probability, correlated Rayleigh fading, energy efficiency.

\section{INTRODUCTION}

W ITH the advent of wireless technologies over the last decade, Wireless Sensor Networks (WSN's) are overtaking wired networks in the field of sensing [1]. Clock synchronization of nodes constitutes a fundamental requirement in WSN since it acts as a reference to data aggregation, localization, energy management. Yet, WSN nodes clock synchronization remains amongst the most challenging open topics in WSN's [2]. As WSN nodes usually count on a limited energy budget, clock synchronization schemes are required to be implemented with optimal energy consumption, enhancing the lifetime of sensing nodes. Thus, a trade-off of energy consumption vs. clock synchronization accuracy must be considered.

There is a number of clock synchronization techniques that exploit received messages from a given sensor node to produce their clock estimation. For example, the Time Flooding Synchronization Protocol (TFSP) [3] employs the one-way

Manuscript received September 19, 2013. The associate editor coordinating the review of this letter and approving it for publication was R. Madan.

This work is a collaboration among the following laboratories: Embedded Systems Laboratory, Faculty of Engineering, University of Buenos Aires (FIUBA), Argentina; Signal Processing \& Communications Laboratory, FIUBA, and CONICET, Argentina; Signals and Systems for Computing Group, Faculty of Engineering, Catholic University, PUCRS, Brazil; and Sensing, Processing \& Communications Research Laboratory, Faculty of Computing, Engineering \& Sciences, Staffordshire University, UK.

P. Briff and A. Lutenberg are with the Embedded Systems Laboratory, Faculty of Engineering, University of Buenos Aires (FIUBA), Argentina. P. Briff is the corresponding author (e-mail: pbriff@fi.uba.ar).

L. Rey Vega is with the Signal Processing \& Communications Laboratory, FIUBA, and CONICET, Argentina.

F. Vargas is with the Signals and Systems for Computing Group, Faculty of Engineering, Catholic University, PUCRS, Brazil.

M. Patwary is with the Sensing, Processing \& Communications Research Laboratory, Faculty of Computing, Engineering \& Sciences, Staffordshire University, UK.

Digital Object Identifier 10.1109/WCL.2013.110713.130668

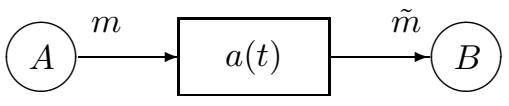

Fig. 1. Node $B$ estimates node $A$ 's clock offset by means of one-way messages.

message exchange mechanism to produces a linear regression estimation of the transmitter's clock offset. Clock estimation accuracy depends on the number of received messages and the degree of accuracy of the retrieval of time stamps [4]. In [5] the authors minimize the outage probability as a function of the transmit power, which does not imply energy optimization; moreover, the authors did not consider the relative velocity of the sensing nodes in their model. The work in [6] studies the trade-off of energy consumption and synchronization accuracy from a local perspective defined by the sleep time of a node, without contemplating the energy spent in the interaction with other nodes. Energy efficiency is defined as a function of payload size in [7] for uncorrelated Rayleigh fading channels. Several works presented in [8] exhibit the requirement of energy minimization in WSN without considering the wireless channel model in the analyses. In this paper we introduce a mathematical model to obtain a trade-off between intended clock synchronization accuracy and required energy, for moving nodes under small-scale Rayleigh fading channels.

\section{Model Statement}

\section{A. Overview}

Let two nodes $A$ and $B$ in the same WSN, as depicted in Fig. 1, require to synchronize with each other by estimating their respective clock offsets via one-way wireless messages exchanged through a correlated Rayleigh fading channel with gain $a(t)$, at time instant $t$. Each node's internal clock can be modelled as $c(t)=\alpha t+\theta$, where $\alpha$ and $\theta$ are the clock skew and offset, respectively [9][10]. The intended achievable synchronization accuracy, denoted by $\epsilon$, is a function of the parameter's estimator and the number of messages employed. Assuming node $A$ sends $m$ number of messages, the expected number of messages successfully received by node $B$, denoted as $\tilde{m}$, depends on the outage probability of the channel, defined as the probability that the received signal quality falls under a minimum acceptable threshold, below which successful signal detection at the receiver node cannot be achieved [11]. Being a function of the transmit power, denoted by $S$, the outage probability will be denoted as $P_{\text {out }}(S)$. Consequently, the pairwise synchronization time, denoted as 
$\delta$, can be defined as follows:

$$
\delta=T_{m} \cdot \frac{m}{\tilde{m}}
$$

where $T_{m}$ is each message's time duration. We propose a mathematical model of the synchronization problem from the pairwise perspective of neighbouring nodes synchronizing with each other, irrespective of network size and topology. To justify the robustness of the proposed model, we also consider additive white Gaussian noise (AWGN) impairments to derive the instantaneous received signal to noise ratio (SNR); power from interferer sources are assumed to be included within noise power denoted as $\sigma_{N}^{2}$.

\section{B. Correlation of Channel Uses}

We consider slow, flat fading channels to study the correlation of the channel uses [12]. The correlated Rayleigh fading channel gain is modelled as a circular complex Gaussian random process [13], defined by:

$$
a(t)=a_{x}(t)+j a_{y}(t)
$$

where $a_{x}(t)$ and $a_{y}(t)$ are mutually uncorrelated zero-mean Gaussian processes. Let $s_{i}(t)=A_{0} e^{j\left(\omega t+\varphi_{i}\right)}$, be a signal of angular frequency $\omega$ and phase $\varphi_{i}$ transmitted through the aforementioned small-scale fading channel, and let $\tilde{s_{i}}(t)$ be the received signal defined by:

$$
\tilde{s}_{i}(t)=\operatorname{Re}\left\{a(t) s_{i}(t)\right\}=\operatorname{Re}\left\{\left|\tilde{r}_{i}(t)\right| e^{j\left(\omega t+\varphi_{i}+\phi_{i}(t)\right)}\right\}
$$

where $i=1, \ldots, m$ is the signal index, and the received complex envelope $\tilde{r}_{i}(t)$ is described by:

$$
\tilde{r}_{i}(t)=X_{i}(t)+j Y_{i}(t)=r_{i} e^{j \phi_{i}(t)}=A_{0} a_{x}(t)+j A_{0} a_{y}(t)
$$

where $r_{i} \triangleq\left|\tilde{r}_{i}(t)\right|$ and $\phi_{i}(t) \triangleq \arg \left(\tilde{r}_{i}(t)\right)$. By defining the transmit power $S \triangleq A_{0}^{2} / 2$, the correlational properties of each component of $\tilde{r}_{i}(t)$ for normalized channel gain are [13]:

$$
R(\tau) \triangleq R_{X X}(\tau)=R_{Y Y}(\tau)=S J_{0}\left(2 \pi \frac{v}{\lambda} \tau\right)
$$

where $J_{0}(\cdot)$ is the zero-order Bessel function of the first kind [14], [12], $v$ the nodes' relative velocity, and $\lambda$ the transmitted signal's wavelength.

\section{Clock Offset Estimation Accuracy as a FUnCTION OF TRANSMIT POWER}

\section{A. Problem To Solve: Energy Optimization}

We use the one-way message exchange mechanism to deal with the energy trade-offs in clock synchronization for WSN, for simplicity reasons. Our model solves the underlying energy optimization problem by approaching it from a statistical theory perspective. Let $\hat{\theta}$ be the estimated clock offset between nodes, with actual clock offset $\theta$, then the estimator's variance $\sigma_{\hat{\theta}}^{2}$ relates with the Fisher Information function, denoted by $I(\theta, \tilde{m})$, as [15]:

$$
\sigma_{\hat{\theta}}^{2}(\tilde{m}) \geq \frac{1}{I(\theta, \tilde{m})}
$$

where $I(\theta, \tilde{m})$ relates with the likelihood function of $\theta$, denoted by $f(\theta, \tilde{m})$, as follows [15]:

$$
I(\theta, \tilde{m}) \triangleq-E\left[\frac{\partial^{2}}{\partial \theta^{2}} \ln f(\theta, \tilde{m})\right]
$$

For Cramer-Rao efficient estimators [15], i.e. those that attain equality in (6), the synchronization accuracy can be expressed as a function of the transmit power, denoted by $S_{\text {opt }}$, as follows:

$$
\text { find } S_{o p t} \text { s.t. } \sigma_{\hat{\theta}}^{2}(\tilde{m})=\frac{1}{I(\theta, \tilde{m})}<\epsilon
$$

Pairwise energy optimization can be achieved by minimizing both transmitter and receiver energy, hence the total pairwise synchronization energy, denoted by $A(S, m, \delta)$, determined by the transmit power $S$ and the total synchronization time $(m$. $\delta)$ during which transmitter and received nodes must remain turned on, is given by:

$$
A(S, m, \delta)=S \cdot m \cdot \delta
$$

Thus, the objective function of the minimization problem is described by:

$$
\text { find } S_{o p t} \text { s.t. }\left.\frac{d A(S, m, \delta)}{d S}\right|_{S=S_{o p t}}=0
$$

\section{B. Joint PDF of the Received Envelope's Components}

When transmitting $m$ number of messages, the sets $\boldsymbol{X}=$ $\left\{X_{i}\right\}_{i=1}^{m}, \boldsymbol{Y}=\left\{Y_{i}\right\}_{i=1}^{m}$ described in (4) exhibit the following joint probability density function (pdf):

$$
f_{\boldsymbol{X Y}}(\boldsymbol{x}, \boldsymbol{y})=\frac{\exp \left(-\frac{1}{2}[\boldsymbol{x}, \boldsymbol{y}]^{T} \cdot \boldsymbol{C}^{-1} \cdot[\boldsymbol{x}, \boldsymbol{y}]\right)}{(2 \pi)^{m}|\boldsymbol{C}|^{1 / 2}}
$$

where $\boldsymbol{x}=\left[\begin{array}{llll}x_{1} & x_{2} \ldots x_{m}\end{array}\right]^{T}, \boldsymbol{y}=\left[\begin{array}{llll}y_{1} & y_{2} \ldots y_{m}\end{array}\right]^{T}$, and

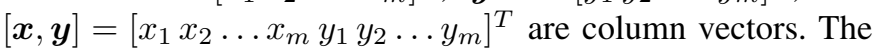
covariance matrix $C$ is defined by:

$$
C=\left(\begin{array}{cc}
C_{a} & 0 \\
0 & C_{a}
\end{array}\right)
$$

being the autocorrelation matrix of both $\boldsymbol{X}$ and $\boldsymbol{Y}$, denoted by $\boldsymbol{C}_{\boldsymbol{a}}$, defined as follows:

$$
\boldsymbol{C}_{\boldsymbol{a}}=\left(\begin{array}{cccc}
\alpha_{11} & \alpha_{12} & \ldots & \alpha_{1 m} \\
\alpha_{12} & \alpha_{11} & \ldots & \alpha_{2 m} \\
\vdots & \vdots & \ddots & \vdots \\
\alpha_{m 1} & \alpha_{m 2} & \ldots & \alpha_{m m}
\end{array}\right)
$$

where $\alpha_{i j}=R\left(T_{s} \cdot(i-j)\right)$ and $1 / T_{s}$ is the symbol rate. The correlation level, denoted by $\rho$, is defined by [5]:

$$
\rho=\frac{\|\boldsymbol{C}-\operatorname{diag}(\boldsymbol{C})\|_{F}}{\|\boldsymbol{C}\|_{F}}
$$

where $\|\boldsymbol{C}\|_{F}$ is the Frobenius norm of $\boldsymbol{C}$ and $\operatorname{diag}(\boldsymbol{C})$ is a matrix containing only the main diagonal of $C$. Hence, $\rho$ ranges from 0 (uncorrelated channel uses) to 1 (highly correlated channel uses).

\section{Received Instantaneous Power}

Recalling (4), the absolute value of the received envelope, $r_{i}$, follows a Rayleigh distribution and the phase, $\phi_{i}(t)$, or simply denoted as $\phi_{i}$ from this point onwards, is uniformly distributed in the range $[0,2 \pi]$, namely $x_{i}=r_{i} \cos \phi_{i}$ and 
$y_{i}=r_{i} \sin \phi_{i}$, with $r_{i} \geq 0$ and $\phi_{i} \in[0,2 \pi]$. Therefore, the joint pdf of the received complex envelopes is defined by:

$$
\begin{aligned}
f_{\boldsymbol{r} \phi}(\boldsymbol{r}, \boldsymbol{\phi}) & =\left|J_{\boldsymbol{X} \boldsymbol{Y}}(\boldsymbol{r}, \boldsymbol{\phi})\right| f_{\boldsymbol{X} \boldsymbol{Y}}(\boldsymbol{x}(\boldsymbol{r}, \boldsymbol{\phi}), \boldsymbol{y}(\boldsymbol{r}, \boldsymbol{\phi})) \\
& =f_{\boldsymbol{X} \boldsymbol{Y}}(\boldsymbol{x}(\boldsymbol{r}, \boldsymbol{\phi}), \boldsymbol{y}(\boldsymbol{r}, \boldsymbol{\phi})) \prod_{i=1}^{m} r_{i}
\end{aligned}
$$

where $\boldsymbol{r}=\left[r_{1} \ldots r_{m}\right]^{\top}, \boldsymbol{\phi}=\left[\phi_{1} \ldots \phi_{m}\right]^{\top}$, and $\left|J_{\boldsymbol{X} \boldsymbol{Y}}(\boldsymbol{r}, \boldsymbol{\phi})\right|$ is the Jacobian of the transformation $\boldsymbol{x}(\boldsymbol{r}, \phi)$ and $\boldsymbol{y}(\boldsymbol{r}, \phi)$. The joint pdf of $\boldsymbol{r}$, denoted by $f_{\boldsymbol{r}}(\boldsymbol{r})$, is obtained as follows:

$$
f_{\boldsymbol{r}}(\boldsymbol{r})=\int_{0}^{2 \pi} \ldots \int_{0}^{2 \pi} f_{\boldsymbol{r} \phi}(\boldsymbol{r}, \boldsymbol{\phi}) d \phi_{1} \ldots d \phi_{m}
$$

Let $\tilde{M}$ be a correlated binomial random variable representing the number of successfully received messages, with expected value $E[\tilde{M} \mid m]=\tilde{m}$. Consider the probability of receiving $k$ successful messages when transmitting $m$ messages, denoted as $P(\tilde{M}=k)$, for $k=0,1, \ldots, m$. The expression of $P(\tilde{M}=k)$ is obtained from joint probability of the envelopes $r_{i}$ with respect to the limit $\beta=\sqrt{2 \sigma_{N}^{2} \gamma_{0}}$, where $\sigma_{N}^{2}$ is the AWGN noise power at the receiver node and $\gamma_{0}$ is the minimum acceptable SNR threshold. Let $U=\left\{r_{1}, r_{2}, \ldots, r_{m}\right\}$ be a set of received envelopes, then let $L=\left\{V_{l}^{n}\right\}$ be a set containing the $k$-order subsets of distinct combinations of $U$, with $q=\left(\begin{array}{c}m \\ k\end{array}\right), n=1,2, \ldots, q$, and $l=1,2, \ldots, k$. Each subset $V_{l}^{n}$ contains $k$ number of elements, denoted by $v_{l}^{n}$. The relative complement of $V_{l}^{n}$ with respect to $U$, denoted as $\overline{V_{l}^{n}}$, contains $(m-k)$ number of elements, denoted as $w_{l}^{n}$. Therefore, $P(\tilde{M}=k)$ can be found as follows:

$$
P(\tilde{M}=k)=\sum_{n=1}^{q} P_{\boldsymbol{r}}\left(V_{l}^{n}>\beta, \overline{V_{l}^{n}}<\beta\right)
$$

where $V_{l}^{n}>\beta=v_{1}^{n}>\beta, \ldots, v_{k}^{n}>\beta$ indicates $k$ successfully received messages, $\bar{V}_{l}^{n}<\beta=w_{1}^{n}<\beta, \ldots, w_{m-k}^{n}<\beta$ correspond to $(m-k)$ unsuccessfully received messages, and $P_{\boldsymbol{r}}$ is the cumulative density function of $\boldsymbol{r}$, obtained by definite integration of (16). The expression of $E[\tilde{M} \mid m]$ is given by:

$$
\tilde{m}=E[\tilde{M} \mid m]=\sum_{k=1}^{m} k P(\tilde{M}=k)=m \cdot\left[1-P_{\text {out }}(S)\right]
$$

where the rightmost term in (18) corresponds to the expectation of the binomial distribution. Hence, the average outage probability $P_{\text {out }}(S)$ can be obtained as follows:

$$
P_{\text {out }}(S)=1-\frac{\tilde{m}}{m}
$$

\section{Gaussian Distribution Function of the Clock Offset}

As per (7), the Fisher Information function requires a likelihood function to be applied. Considering the case of Gaussian distributed likelihood functions [16], for $\tilde{m}$ Gaussian i.i.d observations of $\theta$, their joint pdf is expressed as:

$$
f(\theta, \tilde{m})=\frac{1}{\left(2 \pi \sigma_{V}^{2}\right)^{\tilde{m} / 2}} \exp \left[-\sum_{j=1}^{\tilde{m}} \frac{\left(\theta_{i}-\theta\right)^{2}}{2 \sigma_{V}^{2}}\right]
$$

where $\sigma_{V}^{2}$ is the variance of the measurement noise of $\theta$. Operating with (7), (8) and (20), we obtain:

$$
I(\theta, \tilde{m})=\frac{\tilde{m}}{\sigma_{V}^{2}}>\frac{1}{\epsilon}
$$

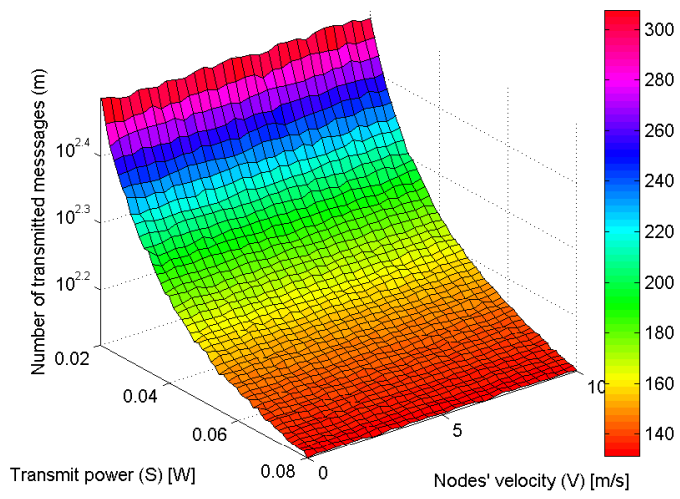

Fig. 2. Number of messages vs. transmit power and nodes' relative velocity. Simulation parameters: $f=2.4 \mathrm{GHz}, T_{m}=3 \mathrm{~ms}, T_{s}=80 T_{m}, \sigma_{N}^{2}=7 \mathrm{~mW}$, $\sigma_{V}=1, \gamma_{0}=5 \mathrm{~dB}, \epsilon=0.01$.

Recalling (9) and taking the lower bound condition in (21), the minimization problem becomes:

$$
\text { minimize } A(S, m, \delta) \text { s.t. } \frac{\tilde{m}}{\sigma_{V}^{2}}=\frac{1}{\epsilon}
$$

By combining (1), (9) and (18) into (22), the minimization problem presented in (10) becomes:

$$
\left.\frac{\sigma_{V}^{2} T_{m}}{\epsilon} \frac{d}{d S}\left\{\frac{S}{\left[1-P_{\text {out }}(S)\right]^{2}}\right\}\right|_{S=S_{\text {opt }}}=0
$$

The solution to (23) determines the minimum transmit power $S_{\text {opt }}$ for achieving the optimal trade-off between energy and synchronization accuracy in WSN's.

\section{Simulation Results}

This section depicts the results of network characteristics given by the transmit power, minimum number of transmitted messages and nodes' relative velocity. Fig. 2 shows the region of energy-optimal network characteristics, which enables the network designer to devise an energy-efficient WSN. The minimum number of transmitted messages $m$ is inversely proportional to the transmit power $S$ for large values of the latter. For small values of $S$ and slow moving nodes, $m$ increases due to the effect of high channel correlation, which is in line with the results presented in [5]. As the velocity grows, the channel correlation decreases and the problem tends to the uncorrelated channel case studied in [4]. Fig. 3 depicts the energy trade-offs and number of transmitted messages for nodes moving at constant velocity, for different values of the synchronization accuracy $\epsilon$. For a given $S$, the synchronization energy grows as $\epsilon$ is enhanced. Fig. 4 compares the proposed lower limit with the results obtained in [5]. It can be seen the total pairwise energy employed by [5] is above the optimal lower bound presented in this work.

\section{Application to WSN Design}

Considering the tracking application of energy-constrained moving nodes detailed in [17], let each node be allocated an energy budget to achieve synchronization, denoted as $E_{\text {sync }}$. Fig. 3 depicts the family of curves that satisfy a 


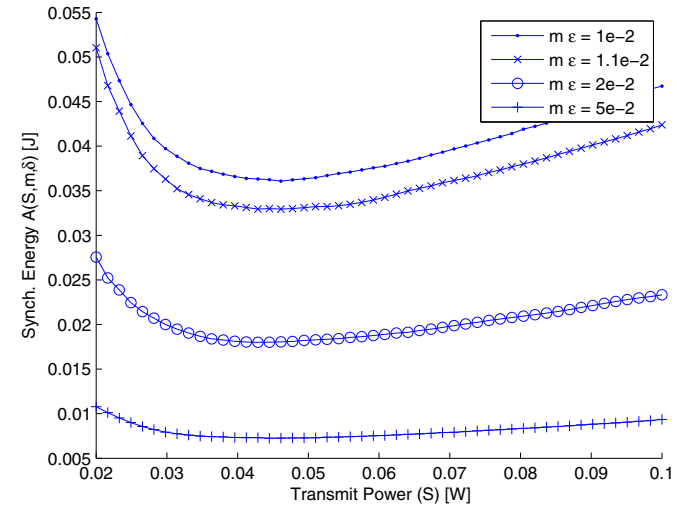

Fig. 3. Total synchronization energy $A(S, m, \delta)$ as a function of $S$. Simulation parameters: $f=2.4 \mathrm{GHz}, v=1 \mathrm{~m} / \mathrm{s}, T_{m}=3 \mathrm{~ms}, T_{s}=80 T_{m}$, $\sigma_{N}^{2}=7 \mathrm{~mW}, \sigma_{V}^{2}=1, \gamma_{0}=5 \mathrm{~dB}$.

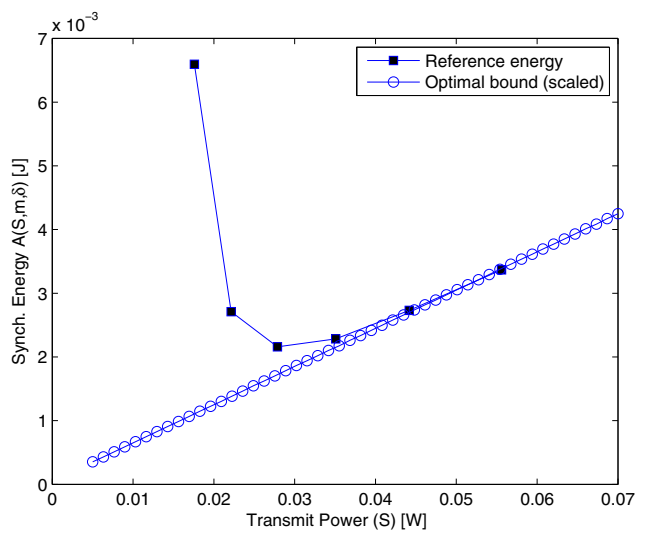

Fig. 4. Comparison of the total synchronization energy $A(S, m, \delta)$ between [5] and the proposed lower bound, as a function of $S$. Simulation parameters: $f=2.4 \mathrm{GHz}, v=1 \mathrm{~m} / \mathrm{s}, T_{m}=3 \mathrm{~ms}, \rho=0.4, \sigma_{N}^{2}=0.13 \mathrm{~mW}$, $\sigma_{V}^{2}=1, \gamma_{0}=5 \mathrm{~dB}, \epsilon=0.05$.

given synchronization energy, for different values of $\epsilon$. Let $E_{\text {sync }}=37 \mathrm{~mJ}, \epsilon=10^{-2}$, and $v=1 \mathrm{~m} / \mathrm{s}$, then for $A(S, m, \delta)=E_{\text {sync }}$, Fig. 3 shows that the optimal energy solution is obtained when $S_{\text {opt }}=45 \mathrm{~mW}$, with $m=150$ as indicated in Fig. 2. The total synchronization time, denoted as $T_{\text {sync }}$, can be obtained as $T_{\text {sync }} \triangleq A(S, m, \delta) / S$; hence, $T_{\text {sync }}=37 \mathrm{~mJ} / 45 \mathrm{~mW} \approx 0.82 \mathrm{~s}$. Furthermore, let us consider time constraints given by the maximum acceptable synchronization time, denoted as $T_{\max }$. Assuming $E_{\text {sync }}=37 \mathrm{~mJ}$ and $T_{\max }=0.5 \mathrm{~s}$, Fig. 3 shows that $A(S, m, \delta)$ can be fixed to meet $T_{\text {sync }} \leq T_{\max }$ within the energy budget by trading $10 \%$ of the synchronization accuracy, i.e. $\epsilon=1.1 \times 10^{-2}$, thus obtaining $S=75 \mathrm{~mW}$ and $m=137$.

\section{CONCLUSIONS}

The proposed model provides a useful tool for the network designer to devise the WSN synchronization deployment that best fits an energy-constrained application with moving nodes under correlated Rayleigh fading channels. This work presents a framework which allows to obtain an energyoptimal solution by finely tuning the transmit power, the number of transmitted messages and the synchronization accuracy within a predefined synchronization energy budget. For timeconstrained and energy-constrained applications, the number of transmitted messages plays a predominant role in the total synchronization time, which is why the synchronization accuracy shall be strategically chosen from a set of solutions made readily available in the proposed model.

\section{REFERENCES}

[1] A. Swami, Q. Zhao, Y. Hong, and L. Tong, Wireless Sensor Networks, Signal Processing and Communications Perspectives. Wiley, 2007, pp. 9-89.

[2] A. Araujo, J. Blesa, E. Romero, and D. Villanueva, "Security in cognitive wireless sensor networks: challenges and open problems." EURASIP J. Wireless Commun. and Networking, vol. 2012, p. 48, 2012.

[3] M. Maroti, B. Kusy, G. Simon, and A. Ledeczi, "The flooding time synchronization protocol," in Proc. 2004 International Conference on Embedded Networked Sensor Systems, pp. 39-49.

[4] P. Briff, F. Vargas, A. Lutenberg, and L. Rey Vega, "On the trade-off of power consumption and time synchronization quality in wireless sensor networks," in Proc. 2012 IEEE Conference on Sensors, pp. 1927-1930, 2012.

[5] L. Li, K. Namuduri, and S. Fu, "Cooperative communication based on random beamforming strategy in wireless sensor networks," in Proc. 2012 IEEE Global Communications Conference, pp. 4108-4113.

[6] G. Deng and F. Zhang, "A power management for probabilistic clock synchronization in wireless sensor networks," in Proc. 2006 IEEE International Conference on Communication Technology, pp. 1-4.

[7] C. Noda, S. Prabh, M. Alves, and T. Voigt, "On packet size and error correction optimisations in low-power wireless networks," in 2013 IEEE International Conference on Sensing, Communication and Networking.

[8] R. B. Araujo, C. H. Ribeiro, D. Turgut, J. Ueyama, and T. Braun, "Deploying real-life WSN applications: challenges, solutions, and future directions," 2013.

[9] F. Ren, C. Lin, and F. Liu, "Self-correcting time synchronization using reference broadcast in wireless sensor network," in IEEE Wireless Commun., 2008.

[10] L. Schenato and G. Gamba, "A distributed consensus protocol for clock synchronization in wireless sensor network," in Proc. 2007 IEEE Conference on Decision and Control.

[11] A. Goldsmith, Wireless Communications. Cambridge University Press, 2005, pp. 24-179.

[12] B. Sklar, "Rayleigh fading channels in mobile digital communication systems-part 1: characterization," IEEE Commun. Mag., pp. 136-146, 1997.

[13] C. Komninakis and R. D. Wesel, "Joint iterative channel estimation and decoding in flat correlated Rayleigh fading," IEEE J. Sel. Areas Commun., vol. 19, no. 9, pp. 1706-1717, 2001.

[14] W. Tang and S. Kassam, "Statistical characteristics of the envelope in diversity combining of two correlated Rayleigh fading channels," IET Commun, vol. 1, no. 3, pp. 405-413, 2007.

[15] S. Kay, Fundamentals of Statistical Signal Processing: Volume 1. Prentice Hall, 1993, pp. 27-77.

[16] K. Noh, Q. Chaudhari, E. Serpedin, and B. Sutter, "Novel clock phase offset and skew estimation using two-way timing message exchanges for wireless sensor networks," IEEE Trans. Commun., vol. 55, no. 4, pp. 766-777, 2007.

[17] K. Römer, "Tracking real-world phenomena with smart dust," in Wireless Sensor Networks. Springer, 2004, pp. 28-43. 